\title{
Testing thermal photon and dilepton rates
}

\section{G. Jackson and M. Laine}

AEC, Institute for Theoretical Physics, University of Bern, Sidlerstrasse 5, CH-3012 Bern, Switzerland

E-mail: jackson@itp.unibe.ch, laine@itp.unibe.ch

ABSTRACT: We confront the thermal NLO vector spectral function (both the transverse and longitudinal channel with respect to spatial momentum, both above and below the light cone) with continuum-extrapolated lattice data (both quenched and with $N_{\mathrm{f}}=2$, at $T \sim 1.2 T_{\mathrm{c}}$ ). The perturbative side incorporates new results, whose main features are summarized. The resolution of the lattice data is good enough to constrain the scale choice of $\alpha_{\mathrm{s}}$ on the perturbative side. The comparison supports the previous indication that the true spectral function falls below the resummed NLO one in a substantial frequency domain. Our results may help to scrutinize direct spectral reconstruction attempts from lattice QCD.

KEYwords: Thermal Field Theory, Lattice QCD, Perturbative QCD, Quark-Gluon Plasma

ARXIV EPRINT: 1910.09567 


\section{Contents}

1 Introduction 1

2 Basic setup 3

3 Theoretical considerations $\quad 4$

3.1 OPE limit 4

3.2 LPM limit 4

3.3 Prediction for IR-singularities around the light cone 5

3.4 Matching of IR-singularities around the light cone 6

3.5 Sum rules 8

4 Comparison with lattice data $\quad 8$

4.1 Summary: resummed spectral functions 8

4.2 Comparison with lattice data for $N_{\mathrm{f}}=0[33] \quad 10$

4.3 Comparison with lattice data for $N_{\mathrm{f}}=2[35,53] \quad 11$

5 Conclusions $\quad 12$

\section{Introduction}

Photons and lepton-antilepton pairs produced in a heavy ion collision are experimentally measurable (cf., e.g., refs. [1-3]) and, given that they do not interact after production, offer for a probe of the inner dynamics of strong interactions in this environment. To leading order in the electromagnetic fine-structure constant $\alpha_{\mathrm{em}}$, the thermal parts of both production rates can be related to the spectral function $\rho_{V}$, associated with the QCD vector current $[4-6]$,

$$
\begin{array}{r}
\frac{\mathrm{d} \Gamma_{\gamma}(k)}{\mathrm{d}^{3} \mathbf{k}}=\frac{\alpha_{\mathrm{em}} n_{\mathrm{B}}(k)}{2 \pi^{2} k} \sum_{i=1}^{N_{\mathrm{f}}} Q_{i}^{2} \rho_{V}(k, k)+\mathcal{O}\left(\alpha_{\mathrm{em}}^{2}\right), \\
\frac{\mathrm{d} \Gamma_{\ell \bar{\ell}}(\omega, k)}{\mathrm{d} \omega \mathrm{d}^{3} \mathbf{k}} \approx \frac{\alpha_{\mathrm{em}}^{2} n_{\mathrm{B}}(\omega)}{3 \pi^{3} M^{2}} \sum_{i=1}^{N_{\mathrm{f}}} Q_{i}^{2} \rho_{V}(\omega, k)+\mathcal{O}\left(\alpha_{\mathrm{em}}^{3}\right) .
\end{array}
$$

Here $n_{\mathrm{B}}$ is the Bose distribution; $M \equiv \sqrt{\omega^{2}-k^{2}}, \omega$ and $k$ are the invariant mass, energy, and momentum, respectively, of a virtual photon; $Q_{i}$ is the charge of a quark of flavour $i$ in units of the elementary charge; disconnected contributions proportional to $\left(\sum_{i} Q_{i}\right)^{2}$ have been omitted; and we have simplified eq. (1.2) by considering energies $2 m_{\ell} \ll M \ll m_{Z}$.

There is a long history of perturbative determinations of $\rho_{V}$ in various kinematic domains. Focussing first on massless quarks, a next-to-leading order (NLO) computation at 
vanishing momentum $(k=0)$ initially suggested that perturbation theory works well [7-9]. However, pushing the energy towards a soft regime $(\omega \ll \pi T, k=0)$ and implementing Hard Thermal Loop (HTL) resummation, a large enhancement was found [10, 11]. Subsequently the focus shifted to the more typical hard momenta $(k \sim \pi T)$, where a logarithmic singularity, shielded by HTL-resummation, was identified when approaching the light cone $(M \ll \pi T)$ [12-14]. In addition, there are non-logarithmic terms of similar magnitude [15], originating from amongst others multiple scatterings with collinear enhancement (the so-called LPM effect [16]), whose systematic handling necessitated a major effort [17-20]. By now these resummed results have been extended up to NLO close to the light cone $[21,22]$. With different methods, the NLO level has also been reached above the light cone $(M \sim \pi T)[23,24]$, and the corresponding results have been shown to permit for a smooth interpolation towards the light-cone ones [25]. Far above the light cone, the spectral function is considerably simpler [26], and can in fact be determined to a high precision [27], by making use of $\mathrm{N}^{4} \mathrm{LO}$ vacuum results [28, 29]. Finally, quark mass effects have been included up to the NLO level at finite temperature, both for $m \gg \pi T$ [30] and for $m \lesssim \pi T$ [31].

Diverse as the progress is, it should be clear that eventually we need to go beyond perturbation theory in the determination of $\rho_{V}$. Lattice QCD entails the measurement of an imaginary-time correlation function $G_{V}(\tau, k)$, which is related to $\rho_{V}$ through

$$
G_{V}(\tau, k)=\int_{0}^{\infty} \frac{\mathrm{d} \omega}{\pi} \rho_{V}(\omega, k) \frac{\cosh \left[\omega\left(\frac{\beta}{2}-\tau\right)\right]}{\sinh \left[\frac{\omega \beta}{2}\right]}, \quad \beta \equiv \frac{1}{T} .
$$

The inversion of this relation is notoriously challenging (cf., e.g., ref. [32]). A recent attempt was made in ref. [33], for continuum-extrapolated quenched QCD. It is clear from eq. (1.3) that, apart from the physical domain $\omega>k$, lattice results are also affected by the spacelike domain $\omega<k$. However it can be argued that, in infinite volume, $\rho_{V}$ should be smooth across the light cone [34]. Thus ref. [33] made use of perturbative information at $M \gtrsim \pi T$ and a fitted interpolating polynomial at $0 \leq \omega \lesssim \sqrt{k^{2}+(\pi T)^{2}}$. A subsequent work considered $N_{\mathrm{f}}=2$ data [35], noting that for the photon channel the contribution of a longitudinal polarization can be subtracted and replacing the interpolating polynomial through a Padé ansatz. Further ideas at implementing analytic continuation have also been put forward [36, 37].

The purpose of the present paper is to scrutinize the spectral reconstructions of refs. [33, 35]. With this aim we improve the status of perturbative predictions in two respects: we incorporate full NLO results for $\omega<k$ [38], and consider separately the transverse and longitudinal polarizations as proposed in ref. [35]. After implementing proper resummation close to light cone, these expressions can be inserted on the right-hand side of eq. (1.3), and subsequently the left-hand side can be compared with lattice data. The perturbative results depend on a parameter, namely the value of the renormalized gauge coupling, and these comparisons permit to "calibrate" the choice made.

Our presentation is organized as follows. In section 2 we define the basic quantities considered. In section 3 we consider various limits, theoretical constraints, and resumma- 
tions that pertain to their perturbative determination. Comparisons with quenched and unquenched lattice data comprise section 4 , whereas conclusions are offered in section 5 .

\section{Basic setup}

Consider the Euclidean vector correlator

$$
\Pi_{\mu \nu}(K) \equiv-\int_{X} e^{i K \cdot X}\left\langle\left(\bar{\psi} \gamma_{\mu} \psi\right)(X)\left(\bar{\psi} \gamma_{\nu} \psi\right)(0)\right\rangle_{T},
$$

where $K=\left(k_{n}, \mathbf{k}\right), X=(\tau, \mathbf{x}),\left\{\gamma_{\mu}, \gamma_{\nu}\right\}=2 \delta_{\mu \nu}$, and $\langle\ldots\rangle_{T}$ denotes a thermal average on a volume with a temporal extent $\tau \in(0, \beta)$. Correspondingly $k_{n}$ is a bosonic Matsubara frequency, viz. $k_{n}=2 \pi n T$, with $n \in \mathbb{Z}$. We denote $K^{2} \equiv k_{n}^{2}+k^{2}$, with $k \equiv|\mathbf{k}|$. An overall minus sign has been inserted in eq. (2.1) for later convenience.

We are mostly interested in a spectral function, which can be obtained as an imaginary part of the Euclidean correlator,

$$
\rho_{\mu \nu}(\mathcal{K})=\operatorname{Im}\left[\Pi_{\mu \nu}(K)\right]_{k_{n} \rightarrow-i\left[\omega+i 0^{+}\right]} .
$$

Its argument is the Minkowskian four-momentum $\mathcal{K} \equiv(\omega, \mathbf{k})$, with $\mathcal{K}^{2} \equiv M^{2}$.

Following ref. [35], we are particularly interested in the linear combinations

$$
\rho_{V} \equiv \rho_{\mu \mu}, \quad \rho_{H} \equiv \rho_{V}+\frac{(D-1) M^{2}}{k^{2}} \rho_{00}
$$

where repeated indices are summed over. Here $D \equiv 4-2 \epsilon$ is the dimension of spacetime. On the light cone, $\rho_{V}$ and $\rho_{H}$ coincide, so that we may replace $\rho_{V}$ through $\rho_{H}$ in eq. (1.1). At leading order (cf., e.g., ref. [39]),

$$
\begin{aligned}
\rho_{\mathrm{V}} & =\frac{N_{\mathrm{c}} M^{2}}{4 \pi k}\left\{2 T\left[l_{1 \mathrm{f}}\left(k_{+}\right)-l_{1 \mathrm{f}}\left(\left|k_{-}\right|\right)\right]+k \theta\left(k_{-}\right)\right\} \\
\rho_{00} & =-\frac{N_{\mathrm{c}}}{12 \pi k}\left\{24 T^{3}\left[l_{3 \mathrm{f}}\left(k_{+}\right)-l_{3 \mathrm{f}}\left(\left|k_{-}\right|\right)\right]+12 k T^{2}\left[l_{2 \mathrm{f}}\left(k_{+}\right)+\operatorname{sign}\left(k_{-}\right) l_{2 \mathrm{f}}\left(\left|k_{-}\right|\right)\right]+k^{3} \theta\left(k_{-}\right)\right\},
\end{aligned}
$$

where we have defined $k_{ \pm} \equiv(\omega \pm k) / 2$ and introduced the polylogarithms

$$
l_{1 \mathrm{f}}(\omega) \equiv \ln \left(1+e^{-\omega / T}\right), \quad l_{2 \mathrm{f}}(\omega) \equiv \operatorname{Li}_{2}\left(-e^{-\omega / T}\right), \quad l_{3 \mathrm{f}}(\omega) \equiv \operatorname{Li}_{3}\left(-e^{-\omega / T}\right) .
$$

Denoting by $g^{2}=4 \pi \alpha_{\mathrm{s}}$ the gauge coupling, by $N_{\mathrm{c}}$ the number of colours, by $C_{\mathrm{F}} \equiv$ $\left(N_{\mathrm{c}}^{2}-1\right) /\left(2 N_{\mathrm{c}}\right)$ the quadratic Casimir coefficient, and by $\mathbb{\&}_{\{P\}}$ a sum-integral with fermionic Matsubara momenta, the NLO expressions for $\Pi_{V} \equiv \Pi_{\mu \mu}$ and $\Pi_{00}$ can be cast in the forms

$$
\begin{aligned}
\Pi_{V}(K)= & 2(D-2) N_{\mathrm{c}} \oiint_{\{P\}}\left[\frac{2}{P^{2}}-\frac{K^{2}}{P^{2}(P-K)^{2}}\right] \\
& +4(D-2) g^{2} N_{\mathrm{c}} C_{\mathrm{F}} \oiint_{\{P Q\}}\left\{\left[\frac{D-2}{P^{4}}-\frac{2}{P^{2}(P-K)^{2}}-\frac{(D-2) K^{2}}{P^{4}(P-K)^{2}}\right]\left[\frac{1}{Q^{2}}-\frac{1}{(Q-P)^{2}}\right]\right. \\
& -\frac{D-4}{Q^{2}(Q-P)^{2}}\left[\frac{1}{P^{2}}-\frac{1}{(P-K)^{2}}\right]-\frac{\frac{1}{2}(D-7) K^{2}}{P^{2}(P-K)^{2} Q^{2}(Q-K)^{2}} \\
& \left.+\frac{(D-6) K^{2}-2(D-2) K \cdot Q}{P^{2}(P-K)^{2} Q^{2}(Q-P)^{2}}+\frac{K^{4}}{P^{2}(P-K)^{2} Q^{2}(Q-K)^{2}(Q-P)^{2}}\right\},
\end{aligned}
$$




$$
\begin{aligned}
\Pi_{00}(K)= & 2 N_{\mathrm{c}} \mathcal{F}_{\{P\}}\left[\frac{2}{P^{2}}-\frac{K^{2}+4 p_{n}\left(p_{n}-k_{n}\right)}{P^{2}(P-K)^{2}}\right] \\
& +4 g^{2} N_{\mathrm{c}} C_{\mathrm{F}} \oint_{\{P Q\}}\left\{(D-2)\left[\frac{1}{P^{4}}-\frac{K^{2}+4 p_{n}\left(p_{n}-k_{n}\right)}{P^{4}(P-K)^{2}}\right]\left[\frac{1}{Q^{2}}-\frac{1}{(Q-P)^{2}}\right]\right. \\
& -\frac{D-4}{Q^{2}(Q-P)^{2}}\left[\frac{1}{P^{2}}-\frac{1}{(P-K)^{2}}\right]-\frac{\frac{1}{2}(D-6)\left(K^{2}-k_{n}^{2}\right)}{P^{2}(P-K)^{2} Q^{2}(Q-K)^{2}} \\
& +\frac{(D-6) K^{2}-2(D-2) K \cdot Q-4(D-4) p_{n} k_{n}+4(D-2) q_{n} k_{n}}{P^{2}(P-K)^{2} Q^{2}(Q-P)^{2}} \\
& \left.+\frac{K^{4}-2 K^{2} k_{n}^{2}-2(D-4) K^{2} p_{n} q_{n}+2(D-2) K^{2} p_{n}^{2}}{P^{2}(P-K)^{2} Q^{2}(Q-K)^{2}(Q-P)^{2}}\right\} .
\end{aligned}
$$

The spectral functions corresponding to all structures here are worked out in ref. [38].

\section{Theoretical considerations}

\subsection{OPE limit}

We now take an imaginary part of eqs. (2.6) and (2.7) according to eq. (2.2). Analytic results can be obtained by considering $|\omega \pm k| \gg \pi T$ [26]. Limiting values for the "master" structures in eq. (2.6) were given in appendix B of ref. [44]. The additional ones appearing in eq. (2.7) can be determined by making use of techniques described in ref. [45], and are listed in ref. [38].

Inserting the expansions, we find that all $1 / \epsilon$-divergences, the corresponding logarithms, as well as thermal corrections proportional to $\int_{p} \frac{n_{\mathrm{B}}}{16 \pi p}$ or $\int_{p} \frac{n_{\mathrm{F}}}{16 \pi p}$, cancel $\left(n_{\mathrm{B}}\right.$ and $n_{\mathrm{F}}$ are the Bose and Fermi distributions, respectively). The remainders read

$$
\begin{aligned}
\rho_{V} & =\frac{N_{\mathrm{c}} M^{2}}{4 \pi}+4 g^{2} C_{\mathrm{F}} N_{\mathrm{c}}\left\{\frac{3 M^{2}}{4(4 \pi)^{3}}+\int_{p} \frac{p}{\pi} \frac{\left(4 n_{\mathrm{F}}-n_{\mathrm{B}}\right)\left(\omega^{2}+\frac{k^{2}}{3}\right)}{3 M^{4}}\right\}+\mathcal{O}\left(\frac{T^{6}}{M^{4}}\right), \\
\rho_{00} & =-\frac{N_{\mathrm{c}} k^{2}}{12 \pi}-4 g^{2} C_{\mathrm{F}} N_{\mathrm{c}}\left\{\frac{k^{2}}{4(4 \pi)^{3}}+\int_{p} \frac{p}{\pi} \frac{\left(4 n_{\mathrm{F}}-n_{\mathrm{B}}\right) k^{2}}{9 M^{4}}\right\}+\mathcal{O}\left(\frac{T^{6}}{M^{4}}\right) .
\end{aligned}
$$

Thereby, in accordance with the general argument in ref. [35], the combination in eq. (2.3) displays only a thermal correction:

$$
\rho_{H}=4 g^{2} C_{\mathrm{F}} N_{\mathrm{c}} \int_{p} \frac{p}{\pi} \frac{4\left(4 n_{\mathrm{F}}-n_{\mathrm{B}}\right) k^{2}}{9 M^{4}}+\mathcal{O}\left(\frac{T^{6}}{M^{4}}\right) .
$$

The integrals evaluate to $\int_{p} \frac{p n_{\mathrm{B}}}{\pi}=\frac{\pi T^{4}}{30}$ and $\int_{p} \frac{p n_{\mathrm{F}}}{\pi}=\frac{7 \pi T^{4}}{240}$, so that $\rho_{H}$ approaches zero from the positive side. We note, however, that the Operator Product Expansion (OPE) shows poor convergence; the actual $\rho_{H}$ switches from negative to positive only around $\omega \sim 20 T$.

\subsection{LPM limit}

We next consider an "opposite" limit to that in section 3.1, namely $M^{2} \rightarrow 0^{ \pm}$. The spatial momentum is kept fixed, with a value $k \sim \pi T$. In this limit the spectral function needs to be resummed in order to account for the Landau-Pomeranchuk-Migdal (LPM) effect. 
Close to the light cone, it is often convenient to represent the two polarizations in a basis different from that in eq. (2.3). Specifically, we define the "transverse" and "longitudinal" spectral functions as

$$
\rho_{T} \equiv \sum_{i \perp \mathbf{k}} \rho_{i i}, \quad \rho_{L} \equiv \rho_{\|}+\rho_{00},
$$

where $\perp$ and $\|$ refer to the components perpendicular and parallel to $\mathbf{k}$. Current conservation implies that $\rho_{L}=-\left(M^{2} / k^{2}\right) \rho_{00}$, and in this basis eq. (2.3) becomes

$$
\rho_{V}=\rho_{T}+\rho_{L}, \quad \rho_{H}=\rho_{T}-(D-2) \rho_{L} .
$$

Following ref. [19], the LPM-resummed spectral functions $\rho_{i}$, with $i=T, L$, read

$$
\begin{aligned}
\left.\rho_{i}\right|_{\mathrm{LPM}} ^{\mathrm{fulll}} \equiv & -\frac{N_{\mathrm{c}}}{\pi} \int_{-\infty}^{\infty} \mathrm{d} \epsilon\left[1-n_{\mathrm{F}}(\epsilon)-n_{\mathrm{F}}(\omega-\epsilon)\right] \\
& \times \lim _{\mathbf{y} \rightarrow \mathbf{0}} \mathbb{P}\left\{\frac{M^{2} \delta_{i, L} \operatorname{Im}[g(\mathbf{y})]}{\omega^{2}}+\frac{\left[\omega^{2}-2 \epsilon(\omega-\epsilon)\right] \delta_{i, T} \operatorname{Im}\left[\nabla_{\perp} \cdot \mathbf{f}(\mathbf{y})\right]}{2 \epsilon^{2}(\omega-\epsilon)^{2}}\right\},
\end{aligned}
$$

where $\mathbb{P}$ stands for a principal value, and $g$ and $\mathbf{f}$ are Green's functions satisfying

$$
\left(\hat{H}+i 0^{+}\right) g(\mathbf{y})=\delta^{(2)}(\mathbf{y}), \quad\left(\hat{H}+i 0^{+}\right) \mathbf{f}(\mathbf{y})=-\nabla_{\perp} \delta^{(2)}(\mathbf{y}) .
$$

The operator $\hat{H}$ acts in the plane transverse to light-like propagation,

$$
\hat{H}=-\frac{M^{2}}{2 \omega}+\frac{\omega\left(m_{\infty}^{2}-\nabla_{\perp}^{2}\right)}{2 \epsilon(\omega-\epsilon)}+i g_{\mathrm{E}}^{2} C_{\mathrm{F}} \int \frac{\mathrm{d}^{2} \mathbf{q}}{(2 \pi)^{2}}\left(1-e^{i \mathbf{q} \cdot \mathbf{y}}\right)\left(\frac{1}{q^{2}}-\frac{1}{q^{2}+m_{\mathrm{E}}^{2}}\right),
$$

where $m_{\infty}^{2}$ is an "asymptotic" quark thermal mass, given in eq. (3.15), whereas $g_{\mathrm{E}}^{2} \simeq g^{2} T$ and $m_{\mathrm{E}}^{2} \simeq g^{2} T^{2}\left(\frac{N_{\mathrm{c}}}{3}+\frac{N_{\mathrm{f}}}{6}\right)$ are parameters of a dimensionally reduced effective theory [40-42].

\subsection{Prediction for IR-singularities around the light cone}

An interesting application of eqs. (3.6)-(3.8) is that by re-expanding them as a power series in $g^{2}$, we can find out what kind of singularities the strict 2-loop results [38] should contain close to the light cone. For this purpose, we follow a procedure described in section 5.1 of ref. [25]. At zeroth order in $g$, the expressions become

$$
\left.\rho_{T}\right|_{\mathrm{LPM}} ^{\left(g^{0}\right)}=\frac{N_{\mathrm{c}} M^{2}}{4 \pi \omega^{3}}\left(\mathcal{I}_{1}-\mathcal{I}_{2}\right),\left.\quad \rho_{L}\right|_{\mathrm{LPM}} ^{\left(g^{0}\right)}=\frac{N_{\mathrm{c}} M^{2}}{4 \pi \omega^{3}} \mathcal{I}_{2}
$$

where

$$
\begin{aligned}
\mathcal{I}_{1} & \equiv\left\{\theta\left(M^{2}\right) \int_{0}^{\omega} \mathrm{d} \epsilon-\theta\left(-M^{2}\right)\left[\int_{-\infty}^{0}+\int_{\omega}^{\infty}\right] \mathrm{d} \epsilon\right\}\left[n_{\mathrm{F}}(\epsilon-\omega)-n_{\mathrm{F}}(\epsilon)\right] \omega^{2} \\
& =\theta\left(M^{2}\right) \omega^{3}+2 \omega^{2} T\left[l_{1 \mathrm{f}}(\omega)-l_{1 \mathrm{f}}(0)\right] \\
\mathcal{I}_{2} & \equiv\left\{\theta\left(M^{2}\right) \int_{0}^{\omega} \mathrm{d} \epsilon-\theta\left(-M^{2}\right)\left[\int_{-\infty}^{0}+\int_{\omega}^{\infty}\right] \mathrm{d} \epsilon\right\}\left[n_{\mathrm{F}}(\epsilon-\omega)-n_{\mathrm{F}}(\epsilon)\right] 2 \epsilon(\omega-\epsilon) \\
& =\theta\left(M^{2}\right) \frac{\omega^{3}}{3}+4 \omega T^{2}\left[l_{2 \mathrm{f}}(\omega)+\operatorname{sign}\left(M^{2}\right) l_{2 \mathrm{f}}(0)\right]+8 T^{3}\left[l_{3 \mathrm{f}}(\omega)-l_{3 \mathrm{f}}(0)\right]
\end{aligned}
$$


The polylogarithms appearing here were defined in eq. (2.5). Even though $\mathcal{I}_{1,2}$ are not analytic around the light cone, eq. (3.9) vanishes there.

Given that the last term in eq. (3.8) is of $\mathcal{O}\left(g^{4}\right)$, the corrections of $\mathcal{O}\left(g^{2}\right)$ are proportional to the parameter $m_{\infty}^{2}$. For $\rho_{L}$, we find no such correction:

$$
\left.\rho_{L}\right|_{\mathrm{LPM}} ^{\left(g^{2}\right)}=0
$$

For $\rho_{T}$, a correction is found which contains a well-known logarithmic divergence as well as a finite part which is discontinuous across the light cone:

$$
\begin{aligned}
\left.\rho_{T}\right|_{\mathrm{LPM}} ^{\left(g^{2}\right)}= & \frac{N_{\mathrm{c}} m_{\infty}^{2}}{2 \pi}\left\{\left[\frac{1}{2}-n_{\mathrm{F}}(\omega)\right]\left(\ln \left|\frac{m_{\infty}^{2}}{M^{2}}\right|-1\right)\right. \\
& +\left[\theta\left(M^{2}\right) \int_{0}^{\omega} \mathrm{d} \epsilon-\theta\left(-M^{2}\right)\left(\int_{-\infty}^{0}+\int_{\omega}^{\infty}\right) \mathrm{d} \epsilon\right] \\
& \left.\times\left[\frac{n_{\mathrm{F}}(\epsilon)-n_{\mathrm{F}}(0)+n_{\mathrm{F}}(\omega-\epsilon)-n_{\mathrm{F}}(\omega)}{\epsilon}+\frac{n_{\mathrm{F}}(\epsilon-\omega)-n_{\mathrm{F}}(\epsilon)}{\omega}\right]\right\} .
\end{aligned}
$$

The integral on the last row is defined in the sense of a principal value at large $|\epsilon|$, where terms $\sim 1 / \epsilon$ cancel due to contributions from negative and positive $\epsilon$. Eq. (3.13) predicts that the strict 2-loop spectral function is discontinuous across the light cone, specifically

$$
\left.\left\{\lim _{\omega \rightarrow k^{+}}-\lim _{\omega \rightarrow k^{-}}\right\} \rho_{T}\right|^{\left(g^{2}\right)}=\frac{g^{2} T^{2} N_{\mathrm{c}} C_{\mathrm{F}}}{8 \pi} \int_{-\infty}^{\infty} \mathrm{d} \epsilon \mathbb{P}\left\{\left[n_{\mathrm{F}}(\epsilon-k)-n_{\mathrm{F}}(\epsilon)\right]\left(\frac{1}{k}-\frac{1}{\epsilon}\right)\right\},
$$

where we inserted the definition of $m_{\infty}^{2}$ from eq. (3.15).

\subsection{Matching of IR-singularities around the light cone}

It is a basic premise of LPM resummation that close to the light cone it eliminates the IR singularities that plague the perturbative series. In other words, when eq. (3.13) is subtracted from the 2-loop expression, the remainder should be non-singular. ${ }^{1}$

The logarithmic singularities and discontinuities originate from two structures, both contained in eq. (2.6). The first source are the factorized terms on the second line. Setting $D \rightarrow 4$ and identifying

$$
m_{\infty}^{2} \equiv g^{2} C_{\mathrm{F}}(D-2) \sum_{\{Q\}}\left[\frac{1}{(Q-P)^{2}}-\frac{1}{Q^{2}}\right] \stackrel{D=4}{=} \frac{g^{2} C_{\mathrm{F}} T^{2}}{4},
$$

the discontinuity from the second line is

$$
\left.\rho_{V}\right|^{\text {disc }} \supset 8 N_{\mathrm{c}} m_{\infty}^{2} \operatorname{Im}\left[\oint_{\{P\}} \frac{1}{P^{2}(P-K)^{2}}\right]_{k_{n} \rightarrow-i\left[\omega+i 0^{+}\right]} .
$$

\footnotetext{
${ }^{1}$ The 2-loop expressions and their IR singularities can also be checked in the regime $\omega, k \ll \pi T$, where they match the imaginary part of the photon HTL self-energy, computed up to NLO in ref. [43].
} 
Carrying out the Matsubara sum and taking the cut, we find

$$
\begin{aligned}
& \operatorname{Im}[\underbrace{}_{\{P\}} \frac{1}{P^{2}(P-K)^{2}}]_{k_{n} \rightarrow-i\left[\omega+i 0^{+}\right]} \\
& \quad=\frac{1}{16 \pi k}\left\{\theta\left(M^{2}\right) \int_{k_{-}}^{k_{+}} \mathrm{d} \epsilon-\theta\left(-M^{2}\right)\left[\int_{-\infty}^{k_{-}}+\int_{k_{+}}^{\infty}\right] \mathrm{d} \epsilon\right\}\left[n_{\mathrm{F}}(\epsilon-\omega)-n_{\mathrm{F}}(\epsilon)\right] .
\end{aligned}
$$

The discontinuity of this expression precisely matches the terms $\propto 1 / k$ in eq. (3.14).

The other terms of eq. (3.14) match the spectral function denoted by

$$
\rho_{\mathcal{I}_{\mathrm{h}}}, \equiv \operatorname{Im}\left[\sum_{\{P Q\}} \frac{2 K \cdot Q}{P^{2}(P-K)^{2} Q^{2}(Q-P)^{2}}\right]_{k_{n} \rightarrow-i\left[\omega+i 0^{+}\right]},
$$

which in ref. [24] was shown to reproduce the logarithmic singularity shown on the first row of eq. (3.13). Here we focus on the discontinuity. The expression obtained after carrying out the Matsubara sums is given in eq. (B.84) of ref. [44], with $\sigma_{1}=\sigma_{2}=\sigma_{4}=-, \sigma_{5}=+$.

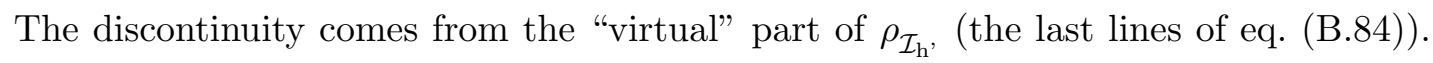
If we define

$$
\begin{aligned}
\Phi\left(\omega, \epsilon_{p}, \mathbf{k} \cdot \mathbf{p}\right) \equiv \int_{\mathbf{q}}[ & \frac{1-n_{\mathrm{F}}\left(\epsilon_{q}\right)+n_{\mathrm{B}}\left(E_{q p}\right)}{4 \epsilon_{q} E_{q p}}\left(\frac{\omega \epsilon_{q}+\mathbf{k} \cdot \mathbf{q}}{\epsilon_{p}+\epsilon_{q}+E_{q p}}+\frac{-\omega \epsilon_{q}+\mathbf{k} \cdot \mathbf{q}}{-\epsilon_{p}+\epsilon_{q}+E_{q p}}\right) \\
& \left.+\frac{n_{\mathrm{F}}\left(\epsilon_{q}\right)+n_{\mathrm{B}}\left(E_{q p}\right)}{4 \epsilon_{q} E_{q p}}\left(\frac{\omega \epsilon_{q}-\mathbf{k} \cdot \mathbf{q}}{\epsilon_{p}-\epsilon_{q}+E_{q p}}+\frac{\omega \epsilon_{q}+\mathbf{k} \cdot \mathbf{q}}{\epsilon_{p}+\epsilon_{q}-E_{q p}}\right)\right],
\end{aligned}
$$

and denote for brevity $\delta_{x} \equiv \delta(x)$, the virtual part reads

$$
\begin{aligned}
& \rho_{\mathcal{I}_{\mathrm{h}}}^{(\mathrm{v})}=\int_{\mathbf{p}} \frac{2 \pi}{4 \epsilon_{p} \epsilon_{p k}} \\
& \times\left\{\Phi\left(\omega, \epsilon_{p}, \mathbf{k} \cdot \mathbf{p}\right)\left[\delta_{\omega-\epsilon_{p}-\epsilon_{p k}}\left[1-n_{\mathrm{F}}\left(\epsilon_{p}\right)-n_{\mathrm{F}}\left(\epsilon_{p k}\right)\right]+\delta_{\omega-\epsilon_{p}+\epsilon_{p k}}\left[n_{\mathrm{F}}\left(\epsilon_{p}\right)-n_{\mathrm{F}}\left(\epsilon_{p k}\right)\right]\right]\right. \\
& \left.-\Phi\left(-\omega, \epsilon_{p}, \mathbf{k} \cdot \mathbf{p}\right)\left[\delta_{\omega+\epsilon_{p}+\epsilon_{p k}}\left[1-n_{\mathrm{F}}\left(\epsilon_{p}\right)-n_{\mathrm{F}}\left(\epsilon_{p k}\right)\right]+\delta_{\omega+\epsilon_{p}-\epsilon_{p k}}\left[n_{\mathrm{F}}\left(\epsilon_{p}\right)-n_{\mathrm{F}}\left(\epsilon_{p k}\right)\right]\right]\right\} .
\end{aligned}
$$

Now, the $\delta$ constraints in eq. (3.20) are equivalent to those emerging from eq. (3.16). Recalling $\epsilon_{p k} \equiv|\mathbf{p}-\mathbf{k}|$, a key observation is that if we approach the light cone from above $\left(\omega \rightarrow k^{+}\right)$, only the first channel contributes, and the contribution emerges from the domain $\epsilon_{p k} \approx k-\epsilon_{p}$, i.e. $\mathbf{p} \| \mathbf{k}$ and $\epsilon_{p}<k$. If we approach the light cone from below $\left(\omega \rightarrow k^{-}\right)$, there is a contribution from the second channel, which emerges from the domain $\epsilon_{p k} \approx \epsilon_{p}-k$, i.e. $\mathbf{p} \| \mathbf{k}$ and $\epsilon_{p}>k$. Below the light cone there is also a contribution from the fourth channel, but now it emerges from the domain $\epsilon_{p k} \approx \epsilon_{p}+k$, i.e. $-\mathbf{p} \| \mathbf{k}$ and $\epsilon_{p}>0$. In total we get

$$
\begin{array}{r}
\left\{\lim _{\omega \rightarrow k^{+}}-\lim _{\omega \rightarrow k^{-}}\right\} \rho_{\mathcal{I}_{\mathrm{h}}}^{(\mathrm{v})}=\frac{1}{8 \pi k} \int_{0}^{\infty} \mathrm{d} \epsilon_{p}\left\{\left[n_{\mathrm{F}}\left(\epsilon_{p}-k\right)-n_{\mathrm{F}}\left(\epsilon_{p}\right)\right] \Phi\left(k, \epsilon_{p}, k \epsilon_{p}\right)\right. \\
\left.+\left[n_{\mathrm{F}}\left(\epsilon_{p}\right)-n_{\mathrm{F}}\left(\epsilon_{p}+k\right)\right] \Phi\left(-k, \epsilon_{p},-k \epsilon_{p}\right)\right\} .
\end{array}
$$


Carrying out the angular integral in eq. (3.19) and setting subsequently $\omega$ and $\mathbf{k} \cdot \mathbf{p}$ to the values required by eq. (3.21), it can be verified that the UV-divergent vacuum term and the IR-sensitive $^{2}$ thermal terms drop out. Moreover, the integral over $q$ yields

$$
\Phi\left(k, \epsilon_{p}, k \epsilon_{p}\right)=-\Phi\left(-k, \epsilon_{p},-k \epsilon_{p}\right)=\frac{k}{2 \epsilon_{p}} \int_{\mathbf{q}} \frac{n_{\mathrm{F}}\left(\epsilon_{q}\right)+n_{\mathrm{B}}\left(\epsilon_{q}\right)}{\epsilon_{q}}=\frac{k T^{2}}{16 \epsilon_{p}} .
$$

Going over to a variable $\epsilon= \pm \epsilon_{p}$ for convenience, we subsequently find

$$
\left\{\lim _{\omega \rightarrow k^{+}}-\lim _{\omega \rightarrow k^{-}}\right\} \rho_{\mathcal{I}_{\mathrm{h}}}^{(\mathrm{v})}=\frac{T^{2}}{128 \pi} \int_{-\infty}^{\infty} \mathrm{d} \epsilon \mathbb{P}\left\{\frac{n_{\mathrm{F}}(\epsilon-k)-n_{\mathrm{F}}(\epsilon)}{\epsilon}\right\}
$$

Multiplying by $-16 g^{2} N_{\mathrm{c}} C_{\mathrm{F}}$ from eq. (2.6), the part $\propto-1 / \epsilon$ of eq. (3.14) is reproduced.

\subsection{Sum rules}

A traditional further constraint on spectral functions is offered by sum rules (cf., e.g., ref. [46] and references therein). Unlike the OPE and LPM limits, the sum rules are sensitive to the complete frequency domain. However, for $\rho_{V}$ they are of limited value, as they require the subtraction of poorly known vacuum parts (containing a dense spectrum of resonances). In contrast, a nice and convergent sum rule can be obtained for $\rho_{H}$ [35]:

$$
\int_{0}^{\infty} \mathrm{d} \omega \omega \rho_{H}(\omega, k)=0 .
$$

We have used our perturbative results in order to test which frequency domain gives a contribution to eq. (3.24). It must be noted that $\rho_{H}$ displays a highly non-trivial structure, changing sign twice: $\rho_{H}$ is positive at $\omega \leq k$, becomes negative at $\omega \gtrsim k$ as is necessary for the cancellation required by eq. (3.24), but then again becomes positive when $|\omega-k| \gg \pi T$, as shown by eq. (3.3). While we have verified that the sum rule is satisfied within numerical uncertainties by our strict 2-loop result and can also be imposed once resummations are included (cf. below), we also see that the asymptotics plays an important role, with the domain $\omega \geq 20 T$ giving a substantial contribution to the absolute value of the integral.

\section{Comparison with lattice data}

\subsection{Summary: resummed spectral functions}

Having discussed various limits and crosschecks of the spectral functions, we are now ready to put together estimates for phenomenological purposes. The full resummed spectral functions $(i \in\{V, H, T, L\})$ are defined as

$$
\left.\left.\rho_{i}\right|_{\mathrm{NLO}} ^{\text {resummed }} \equiv \rho_{i}\right|_{2 \text {-loop }} ^{\text {strict }}+\left(\left.\rho_{i}\right|_{\mathrm{LPM}} ^{\text {full }}-\left.\rho_{i}\right|_{\mathrm{LPM}} ^{\text {expanded }}\right) \times \phi
$$

where $\left.\rho_{i}\right|_{2 \text {-loop }} ^{\text {strict }}$ is from ref. [38]; $\left.\rho_{i}\right|_{\mathrm{LPM}} ^{\text {full }}$ is from section 3.2 ; and $\left.\left.\rho_{i}\right|_{\mathrm{LPM}} ^{\text {expanded }} \equiv \rho_{i}\right|_{\mathrm{LPM}} ^{\left(g^{0}\right)}+\left.\rho_{i}\right|_{\mathrm{LPM}} ^{\left(g^{2}\right)}$ is from section 3.3. The function $\phi$, which should be unity if resummations were implemented

\footnotetext{
${ }^{2}$ It is practical to regularize IR divergences by setting $E_{q p}^{2} \equiv(\mathbf{q}-\mathbf{p})^{2}+\lambda^{2}$, with $\lambda \rightarrow 0$ at the end.
} 

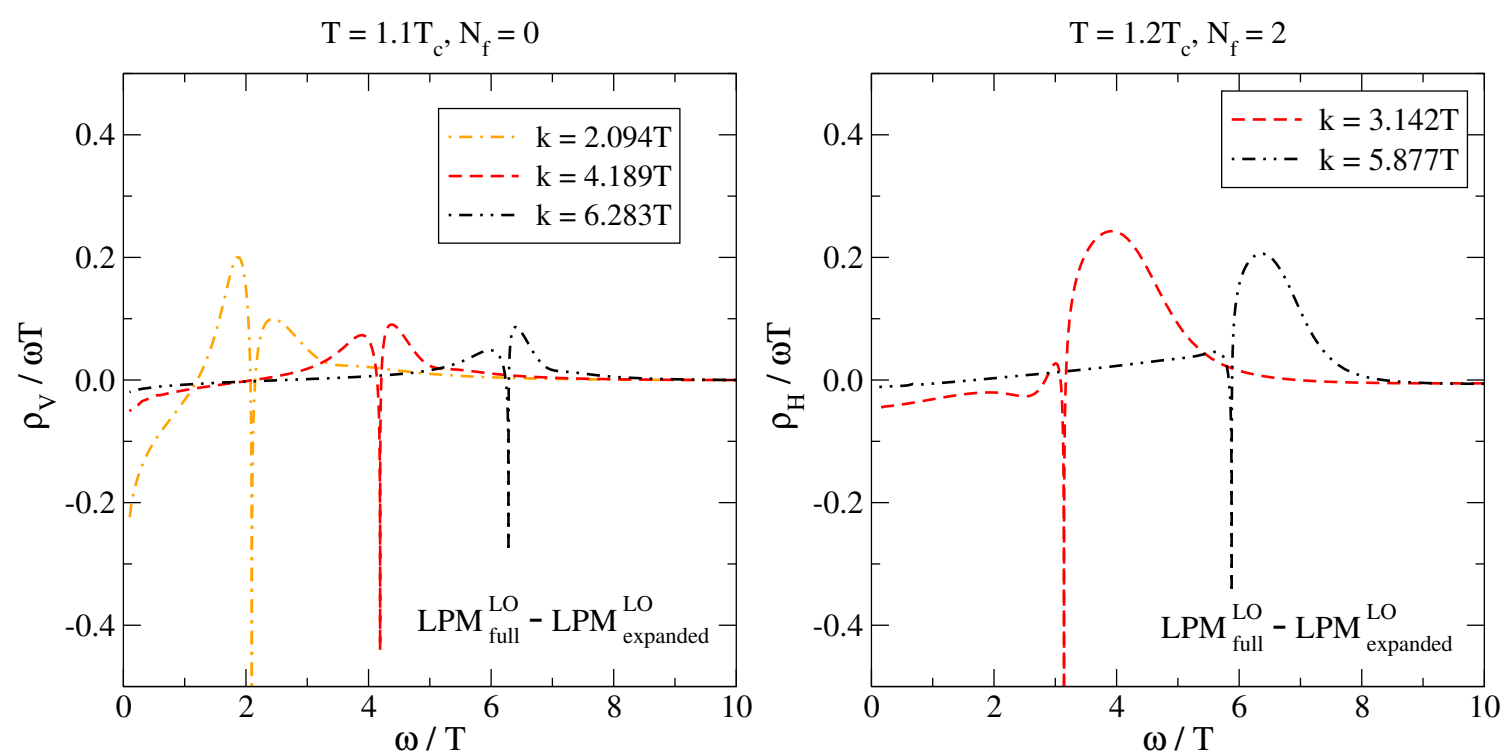

Figure 1. Left: the modification of $\rho_{V}$ (cf. eq. (2.3)) by LPM-resummation (cf. section 3.2), for $\bar{\mu}=\bar{\mu}_{\text {opt }}$. The re-expanded version of the LPM result (cf. section 3.3) has been subtracted in order to avoid double-counting once the result is combined with the full unresummed 2-loop expression, cf. eq. (4.1). The logarithmic singularity cancels in this combination. Right: the same for $\rho_{H}$ (cf. eqs. (2.3), (3.5)).

"exactly", and must in any case equal unity in the IR domain, can be used to correct for the fact that kinematic simplifications pertinent only to the IR domain have been employed in order to implement the resummation. Outside of this domain, we can use $\phi$ to switch off the resummation more rapidly than it would switch off otherwise. We find it practical to define $\phi^{\mathrm{LO}} \equiv \theta\left(\omega^{*}-\omega\right)$, where $\omega^{*}$ is chosen so that the second structure of eq. (4.1) satisfies eq. (3.24) (just like the first structure does). The superscript LO stands for leading-order LPM resummation, as described in sections 3.2 and 3.3, and we find that numerically $\omega^{*} \sim 15 \ldots 25 T$, depending on $k$. We also incorporate NLO LPM-resummed results from ref. [22], however for these the "expanded" version is not available, and we thus impose a faster cutoff away from the light cone, inspired by discussions in ref. [22],

$$
\phi^{\mathrm{NLO}} \equiv \theta(k-\omega) \frac{e^{\omega / T}-1}{e^{k / T}-1}+\theta(\omega-k) \frac{e^{k / T}}{e^{\omega / T}} .
$$

In order to display the practical effect of the resummation, consider the difference $\left.\rho_{i}\right|_{\mathrm{LPM}} ^{\text {full }}-\left.\rho_{i}\right|_{\mathrm{LPM}} ^{\text {expanded }}$ at leading order. Results are shown in figure 1. Prominent features are a logarithmic divergence around light cone, cancelling the one from $\left.\rho_{i}\right|_{2-\text { loop }} ^{\text {strict }}$, as well as the vanishing of the correction when $\omega \rightarrow 0$ or $\omega \rightarrow \infty$ (in figure 1 the spectral function is divided by $\omega)$.

A practical evaluation of the spectral function necessitates a choice of the renormalization scale for the gauge coupling. Motivated by the arguments in ref. [34], we may expect that the physics of the IR domain is represented by a dimensionally reduced description, 
whereby a fastest apparent convergence criterion suggests [47, 48]

$$
\bar{\mu}_{\mathrm{opt}}^{\left(N_{\mathrm{f}}=0\right)}=6.74 T, \quad \bar{\mu}_{\mathrm{opt}}^{\left(N_{\mathrm{f}}=2\right)}=8.11 T .
$$

Away from the IR domain, the scale should be set by virtuality. In order to smoothly interpolate between these two possibilities, we choose

$$
\bar{\mu}_{\mathrm{opt}} \equiv \sqrt{(\xi \pi T)^{2}+\left|M^{2}\right|}
$$

taking $\xi=1$ for $N_{\mathrm{f}}=0$ and a larger $\xi=2$ for $N_{\mathrm{f}}=2$. As these are on the low side compared with eq. (4.3), we vary $\bar{\mu}$ in the range $(1.0 \ldots 2.0) \times \bar{\mu}_{\text {opt }}$, noting that the gauge coupling grows uncontrollably large for $\bar{\mu}=0.5 \bar{\mu}_{\mathrm{opt}}\left(\alpha_{\mathrm{s}}>0.5\right)$. The gauge coupling is solved for from 5-loop evolution [49-51]. We have verified that the results are stable if resorting to lower-order running or modifying the interpolation in eq. (4.4) while keeping the limits at $\pi T \ll|M|$ and $\pi T \gg|M|$ fixed.

At very large $\omega$, we let $\rho_{V}$ continuously cross into vacuum-like $\mathrm{N}^{4} \mathrm{LO}$ perturbative behaviour [27]. Such results can be inserted into eq. (1.3), in order to construct $G_{V}$. For $\rho_{H}$ the vacuum tail is absent, nevertheless the results for $G_{H}$ are quite sensitive to a broad frequency range $0 \leq \omega \lesssim 30 T$.

\subsection{Comparison with lattice data for $N_{\mathrm{f}}=0[33]$}

We start the lattice comparison with the data that were produced and analyzed in ref. [33]. The correlator measured was

$$
G_{V}(\tau, k) \equiv \int_{\mathbf{x}} e^{-i \mathbf{k} \cdot \mathbf{x}}\left\langle\sum_{i=1}^{3} V^{i}(\tau, \mathbf{x}) V^{i}(0)-V^{0}(\tau, \mathbf{x}) V^{0}(0)\right\rangle_{\mathrm{c}},
$$

where $V^{\mu}$ is the (Minkowskian) vector current and $\langle\ldots\rangle_{\mathrm{c}}$ stands for the connected contractions. In the continuum limit this correlator diverges at small $\tau$ and is conveniently normalized to the free result

$$
\frac{G_{\text {norm }, V}}{6 T^{3}} \equiv \pi(1-2 \tau T) \frac{1+\cos ^{2}(2 \pi \tau T)}{\sin ^{3}(2 \pi \tau T)}+\frac{2 \cos (2 \pi \tau T)}{\sin ^{2}(2 \pi \tau T)} .
$$

For scale setting, we use $T_{\mathrm{c}} / \Lambda_{\overline{\mathrm{MS}}} \simeq 1.24$, which has $\sim 10 \%$ uncertainty [52].

Resummed NLO spectral functions $\rho_{V}$ are shown for three momenta in figure 2(left), and the corresponding imaginary-time correlators $G_{V}$ obtained from eq. (1.3) in figure 2(right), where they are also compared with lattice data. Despite the low temperature, we observe a remarkable agreement. On close inspection, the perturbative curves are above the lattice ones, requiring a non-perturbative suppression of $\rho_{V}$. The same qualitative features persist at $T=1.3 T_{\mathrm{c}}$ (not shown), however the difference between the perturbative and lattice results is slightly smaller, as may be expected from a gradually decreasing $\alpha_{\mathrm{s}}$. The conclusions that we draw from these observations are summarized in section 5 . 

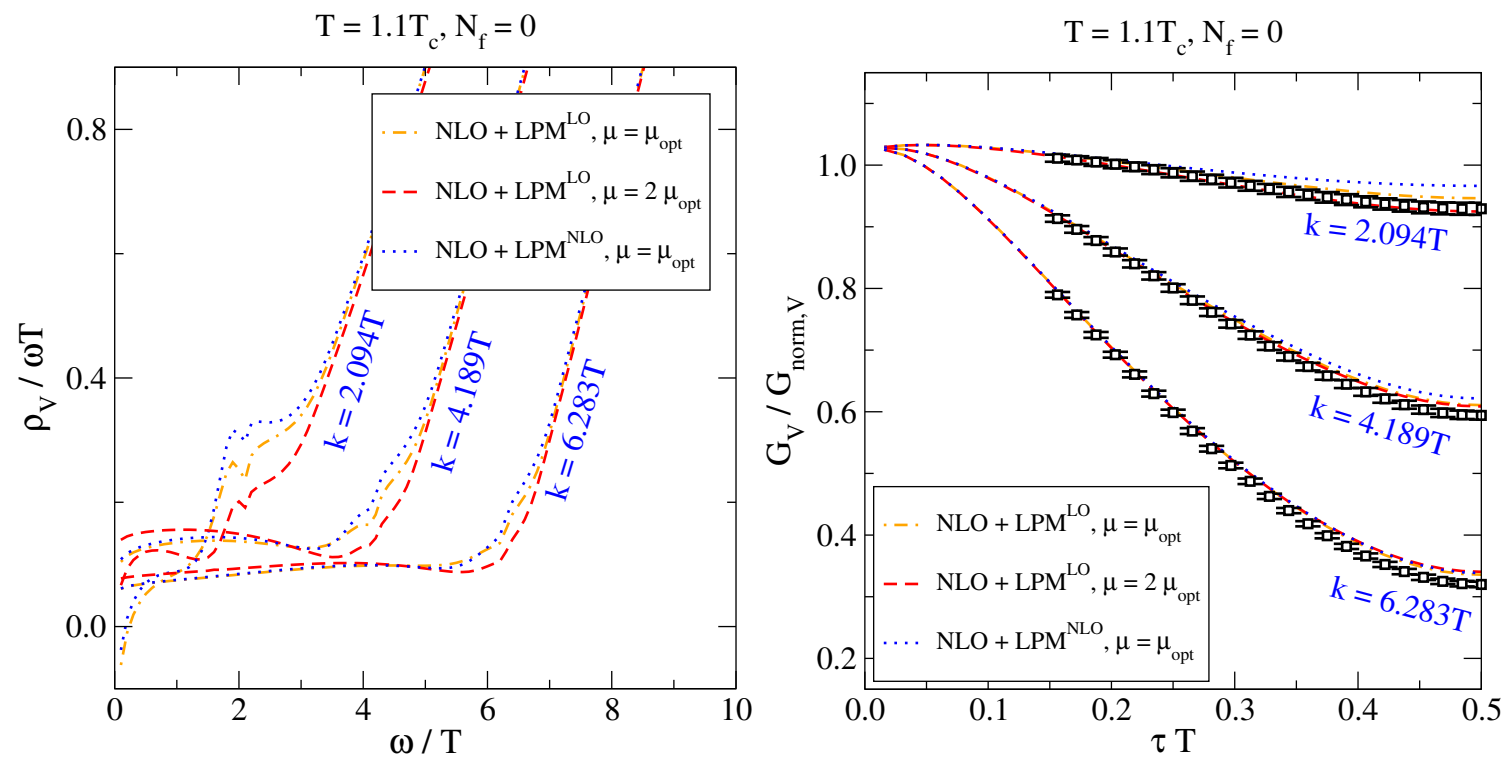

Figure 2. Results for $\rho_{V}$ (left) and $G_{V}$ (right) at $T=1.1 T_{\mathrm{c}}$ for $N_{\mathrm{f}}=0$, the latter normalized to eq. (4.6). $\mathrm{LPM}^{\mathrm{LO}}$ refers to results from sections 3.2 and 3.3, employing the two scale choices $\bar{\mu}=\bar{\mu}_{\text {opt }}$ and $\bar{\mu}=2 \bar{\mu}_{\text {opt }}$ (cf. eq. (4.4)). The notation $\mathrm{LPM}^{\mathrm{NLO}}$ indicates that the contribution from ref. [22] has been added; in this case we use $\bar{\mu}=\bar{\mu}_{\text {opt }}$. The black squares are lattice results from ref. [33]. The spectral function can become negative at very small $\omega$ due to the subtraction of $\rho_{00}$ (cf. eq. (4.5)); the related physics is discussed in more detail around eq. (5.1).

\subsection{Comparison with lattice data for $N_{\mathrm{f}}=2[35,53]$}

Finally we move on to unquenched lattice data, obtained recently for $N_{\mathrm{f}}=2$ in refs. [35, 53]. In this case we concentrate on the ultraviolet finite correlator $\left(\mathbf{k} \equiv k \mathbf{e}_{z}\right)$

$$
G_{H}(\tau, k) \equiv \int_{\mathbf{x}} e^{-i k z}\left\langle\sum_{i=1}^{2} V^{i}(\tau, \mathbf{x}) V^{i}(0)-2\left[V^{z}(\tau, \mathbf{x}) V^{z}(0)-V^{0}(\tau, \mathbf{x}) V^{0}(0)\right]\right\rangle_{\mathbf{c}} .
$$

Let us stress again that the spectral functions corresponding to $G_{V}$ and $G_{H}$ agree on the light cone but are substantially different away from it (cf. figure 2(left) vs. figure 3(left)).

Again a comparison between perturbative and lattice results requires relating physical scales. According to eq. (3.1) of ref. [54], $T_{\mathrm{c}} \simeq 167(25) \mathrm{MeV}$, with units set through $r_{0}=0.503(10) \mathrm{fm}[55]$. Adopting a community average from ref. [56], viz. $r_{0} \Lambda_{\overline{\mathrm{MS}}} \approx$ $0.75(10)$, yields $T_{\mathrm{c}} / \Lambda_{\overline{\mathrm{MS}}} \simeq 0.56$ for $N_{\mathrm{f}}=2$, but with substantial $\sim 25 \%$ uncertainties. For the comparison, a susceptibility is needed as well; we employ the recent continuum extrapolation $\chi=0.88(1) T^{2}$ from ref. [53], consistent with classic expectations [57].

The spectral function $\rho_{H}$ is shown in figure 3(left), and the corresponding imaginarytime correlator $G_{H}$ in figure 3(right). Like in figure 2(right), the lattice correlators fall in general below the perturbative curves. The uncertainties of the perturbative imaginarytime correlators, as reflected by the scale dependence and the difference between LPM $^{\mathrm{LO}}$ and $\mathrm{LPM}^{\mathrm{NLO}}$ resummations, are relatively speaking larger for $N_{\mathrm{f}}=2$, a manifestation of the fact that the dominant vacuum UV tail is absent and therefore the data is more 

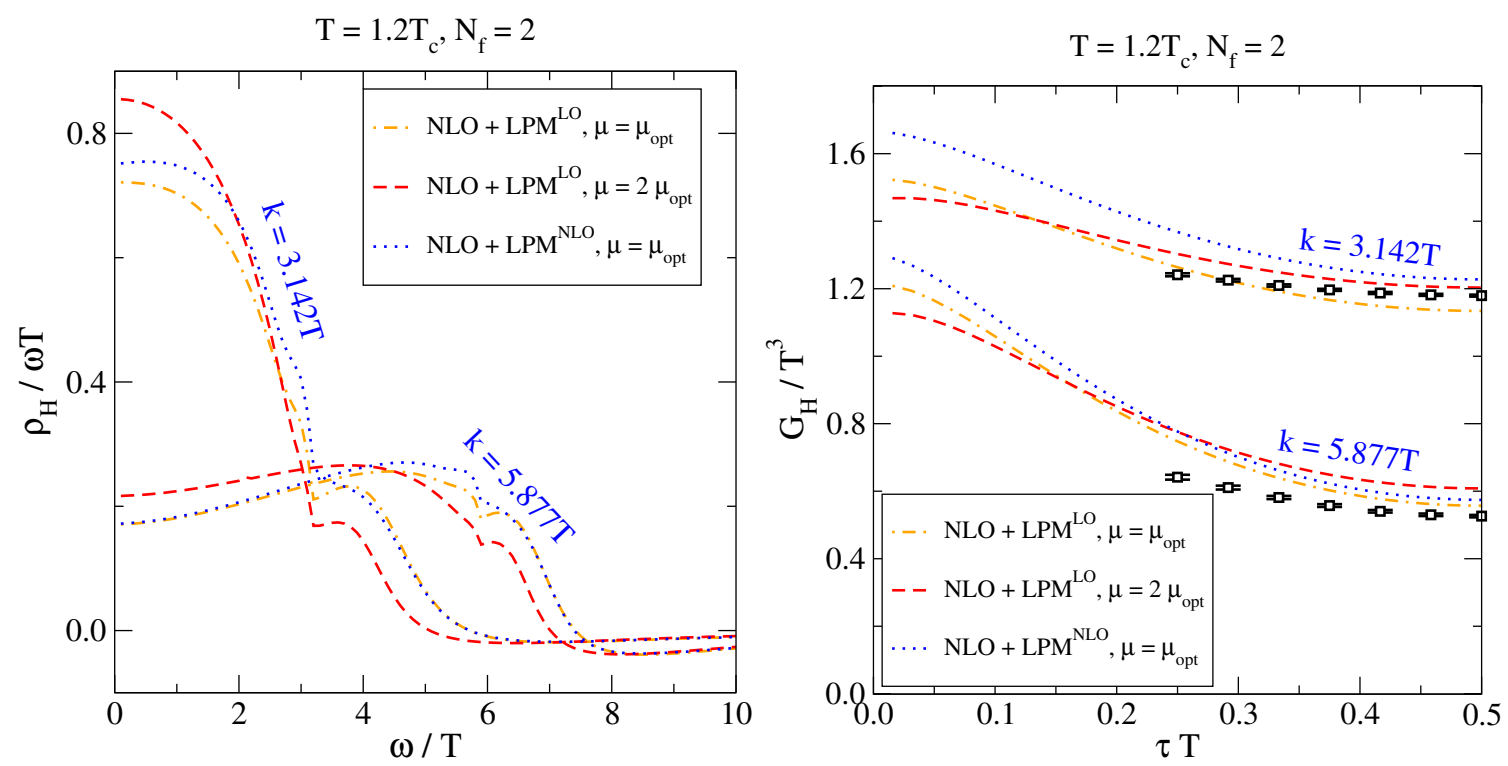

Figure 3. Results for $\rho_{H}$ (left) and $G_{H}$ (right) at $T \simeq 1.2 T_{\mathrm{c}}$ for $N_{\mathrm{f}}=2$. The black squares are lattice data from refs. [35, 53], multiplied by a factor $2 \chi / T^{2}$, where $\chi \approx 0.88 T^{2}$ [53], in order to convert to our units. The notations $\mathrm{LPM}^{\mathrm{LO}}$ and $\mathrm{LPM}^{\mathrm{NLO}}$ and the scale choices are as in figure 2.

sensitive to IR physics. Nevertheless it is comforting that the qualitative pattern remains similar. The conclusions drawn from the comparison are discussed in section 5 .

\section{Conclusions}

Motivated by a comparison with lattice data, unresummed NLO (2-loop) vector spectral functions have recently been extended into two new domains [38]: below the light cone $(\omega<k)$, and to a longitudinal polarization that vanishes at the light cone but is non-zero elsewhere. Even if the spacelike domain, corresponding to deep inelastic scattering off a thermal medium, sounds academic, it is essential for a comparison with lattice data, given that imaginary-time measurements get a large contribution from this region (cf. eq. (1.3)). The longitudinal polarization, in turn, is useful in the UV domain, as it permits to subtract the short-distance singularities from the lattice measurement (cf. eq. (3.3)) [35].

With the 2-loop results at hand, they can be resummed close to the light cone as specified in eq. (4.1) (parametrically, this is needed for $|\omega-k| \lesssim \alpha_{\mathrm{s}} T^{2} / k$ ). Making use of methods developed in ref. [34], this resummation has been worked out to NLO by now $[21,22]$, implying in this context corrections suppressed by $\sqrt{\alpha_{\mathrm{s}}}$. We have incorporated the latter corrections in our results, switching them off away from the light cone when they lose their validity.

The comparison of the imaginary-time correlators following from the resummed NLO spectral functions against lattice data can be viewed as the inspection of many separate "sum rules", one for each $\tau$. Put together, this constrains the spectral function in a nontrivial way. In particular, we find that the correlators are affected by the choice of the 
renormalization scale of $\alpha_{\mathrm{s}}$ (cf. figures 2 and 3). Reasonable agreement is obtained by scale choices reminiscent of those originating from dimensional reduction (cf. eq. (4.3)).

After fixing the renormalization scale, the perturbative results lie in general somewhat above the lattice data. Such a non-perturbative suppression confirms the previous finding based on a polynomial interpolation of $\rho_{V}$ [33]. At the same time the comparison of figures 2(right) and 3(right) testifies to the improved resolution power of the correlator $G_{H}[35]$, so we are looking forward to final results from Padé fits of $\rho_{H}$ [53].

It would be interesting to investigate if resummed NLO rates embedded in hydrodynamical simulations of heavy ion collisions also overshoot the experimental results at small virtualities. To our knowledge this exercise has been implemented only on a rough level so far [58], supporting however this type of an overall trend. Nevertheless, it could still be that the physical photon rate is well predicted or even underestimated by the NLO result, if there is a large suppression of the spectral weigth in some other domain. The general expectation is that strong interactions should suppress thermal fluctuations particularly at small $\omega$ and $k$.

We end by noting that $\rho_{V}$ of the spacelike domain has an interesting relation to the diffusion coefficient of hot QCD matter. For $\omega, k \ll T$, the general theory of statistical fluctuations applies [59] and permits for a "hydrodynamic" prediction (cf., e.g., ref. [60]),

$$
\frac{\rho_{V}(\omega, k)}{\omega} \stackrel{\omega, k \ll T}{\approx} \quad\left(\frac{\omega^{2}-k^{2}}{\omega^{2}+D^{2} k^{4}}+2\right) \chi D
$$

Here $D$ is the diffusion coefficient and $\chi$ is a susceptibility, $\chi \equiv \int_{0}^{\beta} \mathrm{d} \tau \int_{\mathbf{x}}\left\langle V^{0}(\tau, \mathbf{x}) V^{0}(0, \mathbf{0})\right\rangle$. It follows that the zero-frequency $\operatorname{limit} \lim _{\omega \rightarrow 0} \rho_{V}(\omega, k) / \omega$, crosses zero at $k=1 /(\sqrt{2} D)$. The values extracted from our $\left.\rho_{V}\right|_{\mathrm{NLO}} ^{\text {resummed }}$ this way are perfectly consistent with recent lattice estimates $\left(D T \sim 0.2 \ldots 0.8\right.$ at $\left.T=1.1 T_{\mathrm{c}}[61]\right)$ but differ from strict LO perturbative determinations which incorporate further resummations [62] $\left(D T \approx 2.9\right.$ at $\left.T=1.1 T_{\mathrm{c}}[33]\right){ }^{3}$

\section{Acknowledgments}

We thank J. Ghiglieri for discussions and for providing LPM ${ }^{\mathrm{NLO}}$ data from ref. [22], and M. Cè, T. Harris, H.B. Meyer, A. Steinberg and A. Toniato for providing lattice data from refs. $[35,53]$. This project was initiated thanks to a communication by H.B. Meyer, for which we express our gratitude. The work was partly supported by the Swiss National Science Foundation (SNF) under grant 200020B-188712, and by the COST Action CA15213 THOR.

Open Access. This article is distributed under the terms of the Creative Commons Attribution License (CC-BY 4.0), which permits any use, distribution and reproduction in any medium, provided the original author(s) and source are credited.

\footnotetext{
${ }^{3}$ We thank J. Ghiglieri for pointing out that NLO corrections à la ref. [63] do reduce the value of $D T$.
} 


\section{References}

[1] PHENIX collaboration, Dielectron production in Au+Au collisions at $\sqrt{s_{N N}}=200 \mathrm{GeV}$, Phys. Rev. C 93 (2016) 014904 [arXiv:1509.04667] [INSPIRE].

[2] STAR collaboration, Direct virtual photon production in Au+Au collisions at $\sqrt{s_{N N}}=200 \mathrm{GeV}$, Phys. Lett. B 770 (2017) 451 [arXiv: 1607.01447] [INSPIRE].

[3] ALICE collaboration, Direct photon production in $\mathrm{Pb}-\mathrm{Pb}$ collisions at $\sqrt{s_{\mathrm{NN}}}=2.76 \mathrm{TeV}$, Phys. Lett. B 754 (2016) 235 [arXiv: 1509.07324] [INSPIRE].

[4] L.D. McLerran and T. Toimela, Photon and Dilepton Emission from the Quark-Gluon Plasma: Some General Considerations, Phys. Rev. D 31 (1985) 545 [InSPIRE].

[5] H.A. Weldon, Reformulation of finite-temperature dilepton production, Phys. Rev. D 42 (1990) 2384 [INSPIRE].

[6] C. Gale and J.I. Kapusta, Vector dominance model at finite temperature, Nucl. Phys. B 357 (1991) 65 [INSPIRE].

[7] R. Baier, B. Pire and D. Schiff, Dilepton production at finite temperature: Perturbative treatment at order $\alpha_{s}$, Phys. Rev. D 38 (1988) 2814 [INSPIRE].

[8] Y. Gabellini, T. Grandou and D. Poizat, Electron-positron annihilation in thermal QCD, Annals Phys. 202 (1990) 436 [INSPIRE].

[9] T. Altherr and P. Aurenche, Finite Temperature QCD Corrections to Lepton Pair Formation in a Quark-Gluon Plasma, Z. Phys. C 45 (1989) 99 [INSPIRE].

[10] E. Braaten, R.D. Pisarski and T.-C. Yuan, Production of Soft Dileptons in the Quark-Gluon Plasma, Phys. Rev. Lett. 64 (1990) 2242 [InSPIRE].

[11] P. Aurenche, F. Gelis, R. Kobes and E. Petitgirard, Breakdown of the hard thermal loop expansion near the light cone, Z. Phys. C 75 (1997) 315 [hep-ph/9609256] [INSPIRE].

[12] J.I. Kapusta, P. Lichard and D. Seibert, High-energy photons from quark-gluon plasma versus hot hadronic gas, Phys. Rev. D 44 (1991) 2774 [Erratum ibid. D 47 (1993) 4171] [INSPIRE].

[13] R. Baier, H. Nakkagawa, A. Niégawa and K. Redlich, Production rate of hard thermal photons and screening of quark mass singularity, Z. Phys. C 53 (1992) 433 [INSPIRE].

[14] T. Altherr and P.V. Ruuskanen, Low-mass dileptons at high momenta in ultra-relativistic heavy-ion collisions, Nucl. Phys. B 380 (1992) 377 [INSPIRE].

[15] P. Aurenche, F. Gelis, R. Kobes and H. Zaraket, Bremsstrahlung and photon production in thermal QCD, Phys. Rev. D 58 (1998) 085003 [hep-ph/9804224] [INSPIRE].

[16] P. Aurenche, F. Gelis and H. Zaraket, Landau-Pomeranchuk-Migdal effect in thermal field theory, Phys. Rev. D 62 (2000) 096012 [hep-ph/0003326] [INSPIRE].

[17] P.B. Arnold, G.D. Moore and L.G. Yaffe, Photon emission from ultrarelativistic plasmas, JHEP 11 (2001) 057 [hep-ph/0109064] [INSPIRE].

[18] P.B. Arnold, G.D. Moore and L.G. Yaffe, Photon emission from quark gluon plasma: Complete leading order results, JHEP 12 (2001) 009 [hep-ph/0111107] [INSPIRE].

[19] P. Aurenche, F. Gelis, G.D. Moore and H. Zaraket, Landau-Pomeranchuk-Migdal resummation for dilepton production, JHEP 12 (2002) 006 [hep-ph/0211036] [INSPIRE].

[20] M.E. Carrington, A. Gynther and P. Aurenche, Energetic di-leptons from the Quark Gluon Plasma, Phys. Rev. D 77 (2008) 045035 [arXiv:0711.3943] [InSPIRE]. 
[21] J. Ghiglieri, J. Hong, A. Kurkela, E. Lu, G.D. Moore and D. Teaney, Next-to-leading order thermal photon production in a weakly coupled quark-gluon plasma, JHEP 05 (2013) 010 [arXiv: 1302.5970] [INSPIRE].

[22] J. Ghiglieri and G.D. Moore, Low Mass Thermal Dilepton Production at NLO in a Weakly Coupled Quark-Gluon Plasma, JHEP 12 (2014) 029 [arXiv:1410.4203] [INSPIRE].

[23] M. Laine, Thermal 2-loop master spectral function at finite momentum, JHEP 05 (2013) 083 [arXiv: 1304.0202] [INSPIRE].

[24] M. Laine, NLO thermal dilepton rate at non-zero momentum, JHEP 11 (2013) 120 [arXiv: 1310.0164] [INSPIRE].

[25] I. Ghisoiu and M. Laine, Interpolation of hard and soft dilepton rates, JHEP 10 (2014) 083 [arXiv: 1407.7955] [INSPIRE].

[26] S. Caron-Huot, Asymptotics of thermal spectral functions, Phys. Rev. D 79 (2009) 125009 [arXiv:0903.3958] [INSPIRE].

[27] Y. Burnier and M. Laine, Towards flavour diffusion coefficient and electrical conductivity without ultraviolet contamination, Eur. Phys. J. C 72 (2012) 1902 [arXiv:1201.1994] [INSPIRE].

[28] P.A. Baikov, K.G. Chetyrkin and J.H. Kühn, Order $\alpha_{s}^{4} Q C D$ Corrections to $Z$ and $\tau$ Decays, Phys. Rev. Lett. 101 (2008) 012002 [arXiv:0801.1821] [INSPIRE].

[29] P.A. Baikov, K.G. Chetyrkin, J.H. Kühn and J. Rittinger, Adler Function, Sum Rules and Crewther Relation of Order $O\left(\alpha_{s}^{4}\right)$ : the Singlet Case, Phys. Lett. B 714 (2012) 62 [arXiv: 1206.1288] [INSPIRE].

[30] Y. Burnier, M. Laine and M. Vepsäläinen, Heavy quark medium polarization at next-to-leading order, JHEP 02 (2009) 008 [arXiv:0812.2105] [INSPIRE].

[31] Y. Burnier, Quarkonium spectral function in medium at next-to-leading order for any quark mass, Eur. Phys. J. C 75 (2015) 529 [arXiv:1410.1304] [INSPIRE].

[32] H.B. Meyer, Transport Properties of the Quark-Gluon Plasma: A Lattice QCD Perspective, Eur. Phys. J. A 47 (2011) 86 [arXiv:1104.3708] [InSPIRE].

[33] J. Ghiglieri, O. Kaczmarek, M. Laine and F. Meyer, Lattice constraints on the thermal photon rate, Phys. Rev. D 94 (2016) 016005 [arXiv: 1604.07544] [INSPIRE].

[34] S. Caron-Huot, O(g) plasma effects in jet quenching, Phys. Rev. D 79 (2009) 065039 [arXiv:0811.1603] [INSPIRE].

[35] B.B. Brandt, A. Francis, T. Harris, H.B. Meyer and A. Steinberg, An estimate for the thermal photon rate from lattice QCD, EPJ Web Conf. 175 (2018) 07044 [arXiv: 1710.07050] [INSPIRE].

[36] B.B. Brandt, A. Francis, M. Laine and H.B. Meyer, A relation between screening masses and real-time rates, JHEP 05 (2014) 117 [arXiv: 1404.2404] [INSPIRE].

[37] H.B. Meyer, Euclidean correlators at imaginary spatial momentum and their relation to the thermal photon emission rate, Eur. Phys. J. A 54 (2018) 192 [arXiv:1807.00781] [INSPIRE].

[38] G. Jackson, Two-loop thermal spectral functions with general kinematics, arXiv:1910.07552 [INSPIRE]. 
[39] G. Aarts and J.M. Martínez Resco, Continuum and lattice meson spectral functions at nonzero momentum and high temperature, Nucl. Phys. B 726 (2005) 93 [hep-lat/0507004] [INSPIRE].

[40] P.H. Ginsparg, First Order and Second Order Phase Transitions in Gauge Theories at Finite Temperature, Nucl. Phys. B 170 (1980) 388 [inSPIRE].

[41] T. Appelquist and R.D. Pisarski, High-Temperature Yang-Mills Theories and Three-Dimensional Quantum Chromodynamics, Phys. Rev. D 23 (1981) 2305 [InSPIRE].

[42] K. Kajantie, M. Laine, K. Rummukainen and M. Shaposhnikov, Generic rules for high temperature dimensional reduction and their application to the Standard Model, Nucl. Phys. B 458 (1996) 90 [hep-ph/9508379] [INSPIRE].

[43] S. Carignano, M.E. Carrington and J. Soto, The HTL Lagrangian at NLO: the photon case, arXiv: 1909.10545 [INSPIRE].

[44] M. Laine, Thermal right-handed neutrino production rate in the relativistic regime, JHEP 08 (2013) 138 [arXiv: 1307.4909] [INSPIRE].

[45] M. Laine, M. Vepsäläinen and A. Vuorinen, Ultraviolet asymptotics of scalar and pseudoscalar correlators in hot Yang-Mills theory, JHEP 10 (2010) 010 [arXiv:1008.3263] [INSPIRE].

[46] P. Gubler and D. Satow, Finite temperature sum rules in the vector channel at finite momentum, Phys. Rev. D 96 (2017) 114028 [arXiv:1710.02256] [INSPIRE].

[47] S. Huang and M. Lissia, The relevant scale parameter in the high temperature phase of QCD, Nucl. Phys. B 438 (1995) 54 [hep-ph/9411293] [INSPIRE].

[48] M. Laine and Y. Schröder, Two-loop QCD gauge coupling at high temperatures, JHEP 03 (2005) 067 [hep-ph/0503061] [INSPIRE].

[49] P.A. Baikov, K.G. Chetyrkin and J.H. Kühn, Five-Loop Running of the QCD coupling constant, Phys. Rev. Lett. 118 (2017) 082002 [arXiv:1606.08659] [INSPIRE].

[50] F. Herzog, B. Ruijl, T. Ueda, J.A.M. Vermaseren and A. Vogt, The five-loop beta function of Yang-Mills theory with fermions, JHEP 02 (2017) 090 [arXiv:1701.01404] [INSPIRE].

[51] T. Luthe, A. Maier, P. Marquard and Y. Schröder, The five-loop Beta function for a general gauge group and anomalous dimensions beyond Feynman gauge, JHEP 10 (2017) 166 [arXiv: 1709.07718] [INSPIRE].

[52] A. Francis, O. Kaczmarek, M. Laine, T. Neuhaus and H. Ohno, Critical point and scale setting in SU(3) plasma: An update, Phys. Rev. D 91 (2015) 096002 [arXiv:1503.05652] [INSPIRE].

[53] M. Cè, T. Harris, H.B. Meyer and A. Steinberg and A. Toniato, in preparation.

[54] B.B. Brandt, A. Francis, H.B. Meyer, O. Philipsen, D. Robaina and H. Wittig, On the strength of the $U_{A}(1)$ anomaly at the chiral phase transition in $N_{f}=2 Q C D, J H E P 12$ (2016) 158 [arXiv: 1608.06882] [INSPIRE].

[55] P. Fritzsch et al., The strange quark mass and Lambda parameter of two flavor QCD, Nucl. Phys. B 865 (2012) 397 [arXiv: 1205.5380] [INSPIRE].

[56] Flavour Lattice Averaging Group collaboration, FLAG Review 2019, arXiv: 1902.08191 [INSPIRE]. 
[57] A. Vuorinen, Quark number susceptibilities of hot QCD up to $g^{6} \ln g$, Phys. Rev. D 67 (2003) 074032 [hep-ph/0212283] [INSPIRE].

[58] Y. Burnier and C. Gastaldi, Contribution of next-to-leading order and Landau-Pomeranchuk-Migdal corrections to thermal dilepton emission in heavy-ion collisions, Phys. Rev. C 93 (2016) 044902 [arXiv:1508.06978] [InSPIRE].

[59] E.M. Lifshitz and L.P. Pitaevskii, Statistical Physics, Part 2, §88-89.

[60] J. Hong and D. Teaney, Spectral densities for hot QCD plasmas in a leading log approximation, Phys. Rev. C 82 (2010) 044908 [arXiv: 1003.0699] [INSPIRE].

[61] H.-T. Ding, O. Kaczmarek and F. Meyer, Thermal dilepton rates and electrical conductivity of the QGP from the lattice, Phys. Rev. D 94 (2016) 034504 [arXiv: 1604.06712] [INSPIRE].

[62] P.B. Arnold, G.D. Moore and L.G. Yaffe, Transport coefficients in high temperature gauge theories. 2. Beyond leading log, JHEP 05 (2003) 051 [hep-ph/0302165] [INSPIRE].

[63] J. Ghiglieri, G.D. Moore and D. Teaney, QCD Shear Viscosity at (almost) NLO, JHEP 03 (2018) 179 [arXiv: 1802.09535] [INSPIRE]. 